\title{
Simultaneous Melatonin Administration Effectively Deprograms the Negative Influence of Neonatal Hypothyroidism on Immature Follicles but not on Mature Follicles and Body and Ovarian Weights
}

\author{
B.P Thakkara, V.M Zala ${ }^{\mathrm{a}}$, A.V Ramachandran ${ }^{\mathrm{a}, \mathrm{b}}$
}

\begin{abstract}
Background: The impact of simultaneous melatonin administration on neonatal progmming by hypothyroidism (HPOT + MT) on adult ovarian functions and serum hormone profiles has been evaluated in the Charles foster strain of rats.

Methods: The female neonates were rendered hypothyroid (HPOT) by feeding lactating mothers with 6-propyl 2-thiouracil (PTU) through drinking water and were simultaneously administered with melatonin (MT) $(40 \mu \mathrm{g} / \mathrm{animal} / \mathrm{day})$ from day 0 to day 21 in the evening at 16:30 hours just prior to lights off.

Results: The experimental animals (HPOT + MT) consistently showed decreased body and ovary weights compared to controls. The total number of follicles was significantly lower in treated animals (HPOT + MT) but was greater than the HPOT group of animals, and both showed significantly decreased number of follicles compared to control rats. There was a significant reduction in the number of antral follicles and corpora lutea and decreased atretic follicles in experimental rats.
\end{abstract}

Conclusions: It can be concluded from the above results that mela-

Manuscript accepted for publication December 2, 2011

${ }^{\mathrm{a}}$ Division of Reproductive Biology and Endocrinology, Department of Zoology, Faculty of Science, M. S. University of Baroda, Vadodara Gujarat, India

${ }^{\mathrm{b}}$ Corresponding author: A.V Ramachandran.

Email: avrcn@rediffmail.com

doi:10.4021/jem54w tonin is able to nullify the negative influence of hypothyroidism on adult ovarian functions and steroidogenesis to a greater extent.

Keywords: Ovary; Rats; Melatonin; Hypothyroid

\section{Introduction}

Sexual development and reproductive maturation is a protracted event initiated during the foetal stage and influenced by the developing hypothalamic-pituitary-gonadal axis (HPG) [1]. Thyroid hormones are critically involved in embryonic and foetal development of vertebrates [2]. Both thyroid hormone deficiency and excess are known to affect the development and functions of reproductive system $[3,4]$. Prepubertal hypothyroidism induced from the day of birth till day 40 postpartum shows reduced body and ovarian weights, with the ovaries of such rats containing more secondary and less antral follicles, smaller non-atretic antral follicles and more atretic follicles with no corpora lutea compared to age-matched controls [5]. Many studies on postnatal hypothyroidism have reported retarded growth and physical development, delay in eye opening and teething, slow to respond to general environment and also to have depressed body weight [6-11]. Perturbation in endocrine signals in developmentally critical periods like the foetal and neonatal stages can bring about physiological alteration in organ systems within a range of phenotypic plasticity from the same genotype. Such perturbations in endocrine signals in developmentally critical periods are potentially capable of establishing altered adult structural and functional status of organs and, even, impact health positively or negatively by inducing changes in gene transcription, modification of metabolic rates in target cells, changes in cell number or even by bringing about subtle changes in receptor type and number [12]. Previous study on programming by induced thyroid hormone deficiency during the neonatal period in the Charles foster strain of rats has recorded increased follicular atresia and reduced corpora lutea together with decreased secretion of estrogen 
and progesterone from the adult ovary as part of adult plasticity changes [13].

In terms of melatonin status, immature rats have been shown to be relatively more responsive than adults. Administration of melatonin reportedly retards development of both male and female reproductive systems [14-18]. Postnatal days 20 - 40 have been shown to be more critical in this inhibitory effect $[19,20]$. Since the influence of melatonin on the development of the reproductive system has been shown to commence during the prenatal period and extend into the postnatal life [21], melatonin infusion either in the evening or in the morning in the infantile to prepubertal period (10 - 25 days) has been tested in this laboratory. This study showed decreased body weight and testes weight in the period immediately after melatonin treatment more pronouncedly in the evening schedule [22]. In continuation, neonatal programming by melatonin was studied in terms of adult plasticity changes on adult ovarian functions, which revealed increased numbers of various follicles and more than doubled number of corpora lutea with fewer atretic follicles and greater fecundity [23].

Since postnatal programming by hypothyroidism and hypermelatonemia has shown opposite set of effects, an attempt is made in the present study to evaluate the effect of simultaneous neonatal hypothyroidism and hypermelatonemia on adult ovarian functions.

\section{Materials and Methods}

\section{Animals and maintenance}

Healthy female laboratory rat neonates (Charles foster strain) were used for the present study. The animals were maintained at the Sarabhai Research Center, with a constant temperature range of $21 \pm 2{ }^{\circ} \mathrm{C}$ and under a lighting regimen of LD 8:16 throughout the experimental period of study. The animals were fed with standard diet (Amrut Rat Feed) and water ad libitum. The treatment was initiated on day ' 0 ' (day of birth) and terminated on day 21 postpartum. The studies were conducted as per the CPCSEA guidelines and clearance by the ethical committee (Approval No. 827/ac/04/ CPCSEA).

\section{Experimental protocol}

The experimental setup was divided broadly into two groups.

\section{Group I control (C)}

Rat neonates (female) maintained in the laboratory till day 90 served as controls. This consisted of 2 subgroups (as follows) of 10 animals each: (i) Control rats [Maintained as such]; (ii) Experimental rats [Injected i.p. with vehicle $(0.9 \%$ saline) in evening at 16:30 hours].

\section{Group II Hypothyroidism + Melatonin (HPOT + MT)}

Rat neonates (females) subjected to transient hypothyroidism (HPOT) by feeding mothers with $0.1 \%$ 6-propyl 2 -thiouracil (PTU) in drinking water from day 0 to day 21 post partum and simultaneously injected with $40 \mu \mathrm{g}$ melatonin/ animal/day i.p. [N-Acetyl-5- hydroxytryptamine (MT) procured from Sigma Chemical Co. USA] at 16:30 hours consisting of 10 animals each.

\section{Parameters and methods of evaluation}

The treatment was discontinued from day 22 and the animals were sacrificed at 22, 45 and 90 days of age, and various morphometric, gravimetric and histocytometric studies were carried out. The animals were sacrificed under mild anesthesia and blood was collected by brachial venipuncture in centrifuge tubes. They were centrifuged at $4000 \mathrm{rpm}$ and serum was collected and stored at $-4{ }^{\circ} \mathrm{C}$. Later, these serum samples were utilized for assay of various hormones. The viscera was cut open and the ovaries excised, blotted free of tissue fluids and weighed accurately in a Mettler balance. The absolute weights so obtained were converted to relative weights and expressed as percentage of body weight. The ovaries were fixed in Bouin's fluid and processed for paraffin wax histology.

\section{Histology and histometry}

Ovaries were fixed immediately in Bouin's fluid and processed for histological studies. Paraffin sections of $3 \mu \mathrm{m}$ thickness were cut on a microtome and stained with Haematoxylin-Eosin (HE). For morphometry and enumeration of ovarian follicles, homologous cross sections of entire ovary showing better area of vision were chosen. The section area was calculated by integrating the area inside the traced perimeter and volume was calculated by multiplying by the section thickness. The section volume was multiplied by 10 (to account for the number of sections skipped) to give the "10-section" volume; and sum of all the 10-section volumes was obtained to estimate the total ovarian volume (in $\mathrm{mm}^{3}$ ) $[24,25]$. Total counts of different types of follicles were made using an occulometer.

\section{Hormone assays}

The blood for hormone assays was collected from the brachial vein under mild anesthesia before sacrificing the animals. $\mathrm{T}_{3}$ and $\mathrm{T}_{4}$ were assayed by ELISA using kit purchased from Glaxo (product code $\mathrm{H}-\mathrm{T}_{3} \mathrm{H}-0010$ and $\mathrm{H}-\mathrm{T}_{4} \mathrm{H}-0010$ ) and expressed in $\mathrm{ng} / \mathrm{mL}$ of serum. Estradiol and progesterone were assayed by using ELISA kit purchased from General Bio- 

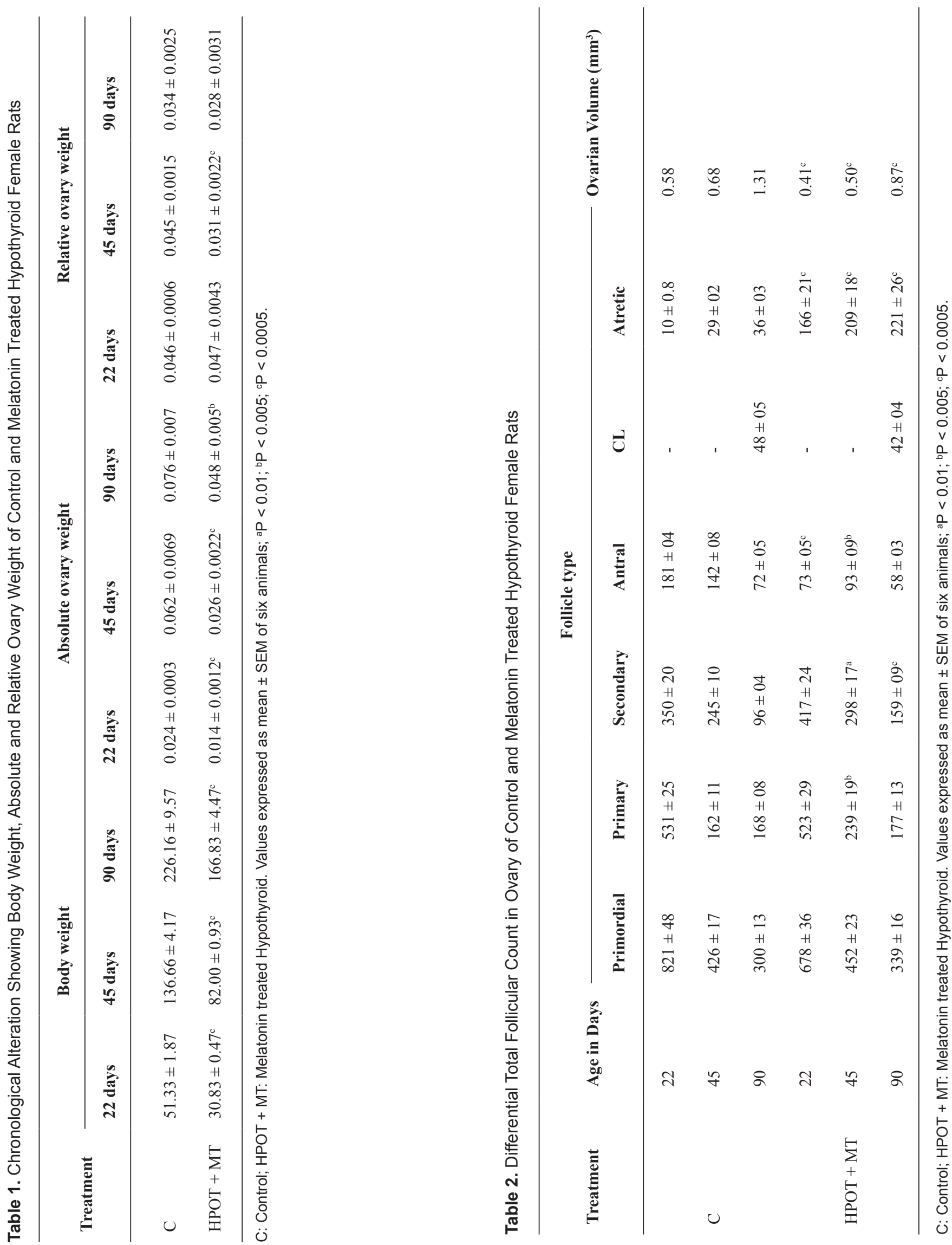
1

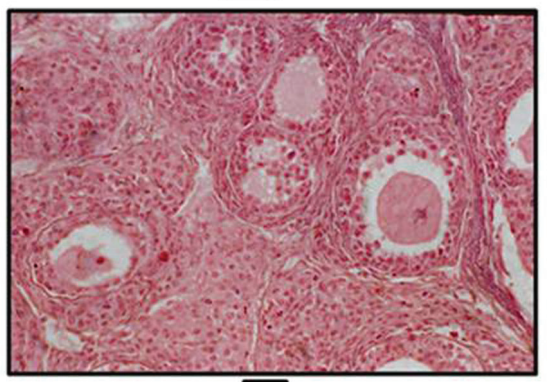

3

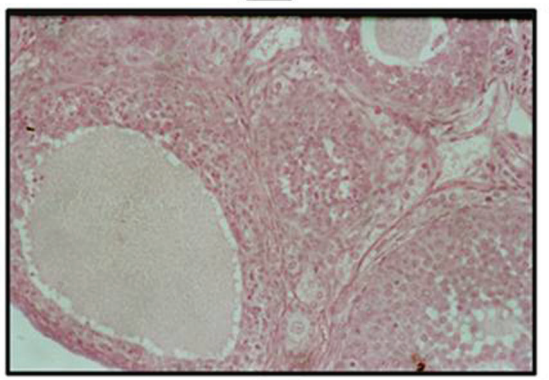

5

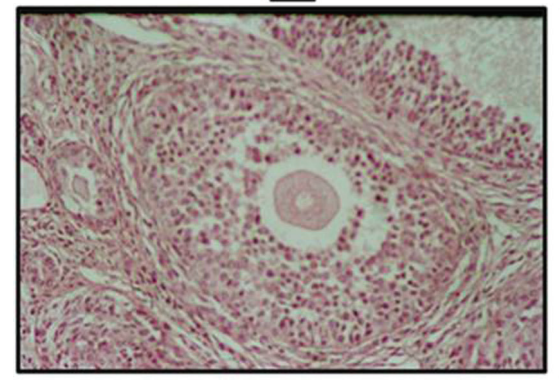

2

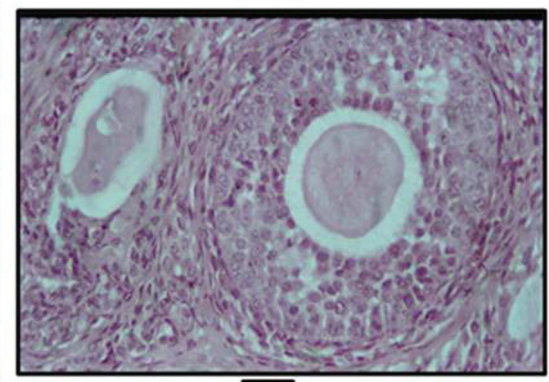

4

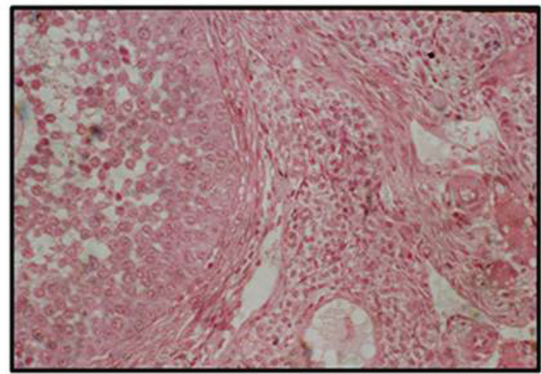

6

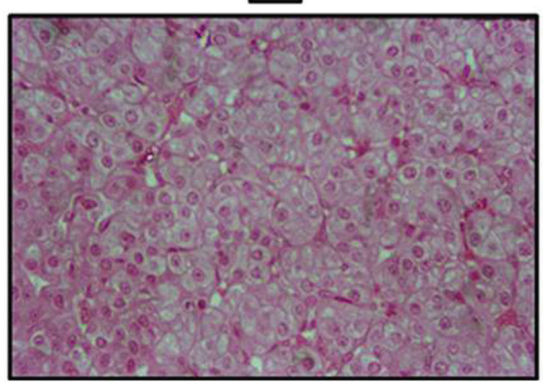

Figure 1. Photomicrographs of sections of ovary of control and melatonin treated hypothyroid rats. Sections of ovaries of 2245 and 90 day old rats $(1,3,5)$ showing less number of atretic follicles and number of primary, secondary and tertiary follicles compared to age matched melatonin treated hypothyroid rats $(2,4,6)$.

logicals Corp, Taiwan and expressed as $\mathrm{ng} / \mathrm{mL}$.

\section{Statistical analysis}

All data are expressed as mean \pm SEM. The data were analyzed by student's t test and two-way analysis of variance (ANOVA) wherever applicable, at 95\% confidence limit.

\section{Results}

\section{Body and ovarian weights}

The body and ovarian weights of experimental rats were consistently lower at all ages of study from 22 - 90 days postpartum. Whereas the body weight at 90 days was $26 \%$ lesser than controls, the absolute and relative weights of ova- ry were respectively $37 \%$ and $18 \%$ lesser (Table 1).

\section{Ovarian histology and histometry}

The ovarian volume in HPOT + ML rats was significantly lower throughout, with a $34 \%$ decrement in the adult stage at 90 days. There was a generalized increase in the total number of follicles at 45 and 90 days. The increase in follicular number was mostly of primordial and secondary follicles but with significant reduction in antral follicles and corpora lutea. The number of atretic follicles was significantly high in the experimental rats (Fig. 1; Table 2).

\section{Serum hormone profile}

Both estrogen and progesterone titers were higher in the immediate post-treatment period; however, their levels were 
Table 3. Hormonal Status of Control and Melatonin Treated Hypothyroid Female Rats

\begin{tabular}{|c|c|c|c|c|c|c|}
\hline \multirow{2}{*}{ Treatment } & \multicolumn{3}{|c|}{ Estradiol } & \multicolumn{3}{|c|}{ Progesterone } \\
\hline & 22 days & 45 days & 90 days & 22 days & 45 days & 90 days \\
\hline $\mathrm{C}$ & $2.04 \pm 0.31$ & $7.83 \pm 0.75$ & $13.07 \pm 1.55$ & $4.75 \pm 0.68$ & $21.75 \pm 2.39$ & $31.59 \pm 4.36$ \\
\hline HPOT + MT & $3.10 \pm 0.92$ & $5.68 \pm 0.52$ & $9.39 \pm 1.84$ & $7.80 \pm 0.35^{\mathrm{c}}$ & $21.75 \pm 1.39$ & $20.27 \pm 3.47$ \\
\hline
\end{tabular}

C: Control; HPOT + MT: Melatonin treated Hypothyroid. Values expressed as mean \pm SEM of six animals; ${ }^{\mathrm{P}}<0.01$; ${ }^{\mathrm{b}} \mathrm{P}$ $<0.005$; $^{\mathrm{c} P}<0.0005$.

significantly lower than the control level at 45 and 90 days. Except for the 22nd day, $\mathrm{T}_{3}$ and $\mathrm{T}_{4}$ levels were significantly higher at all ages in the experimental rats compared to controls. These changes in hormone profile are shown in Table 3,4 .

\section{Discussion}

The results of the present study clearly reveal differential effects of both melatonin and hypothyroidism. Body weight, ovarian weight and volume are all significantly less compared to controls, very much comparable with animals rendered hypothyroid neonatally. Adult HPOT + MT rats show $26 \%$ deficit in the body weight and $18 \%$ deficit in ovarian weight as against $36 \%$ and $21 \%$ deficit respectively in HPOT rats [13]. Clearly, simultaneous melatonin administration is not able to deprogram the hypothyroidism-induced retardation in body and ovarian weights. A distinct sex difference is inferable as both HPOT [26] and HPOT + MT (unpublished) males showed increased testis size as against decreased ovarian weight seen in females herein. Apparently, as the testes respond favourably, the ovaries respond unfavourably to neonatal hypothyroidism in terms of adult gonadal weight. Thyroid hormone seems to be a crucial hormone required for both body and ovary growth in females, while in males the hypothyroidism induced testis enlargement is related with prolonged phase of Sertoli cell proliferation coupled with delayed differentiation [26-28]. Further, while melatonin administration had a potentiating influence on hypothyroidism in males in terms of Sertoli cell proliferation [26], the same in females could not reverse the retardatory influence of hypothyroidism on ovarian growth.

Previous studies showed a marginal increase in the total number of follicles under HPOT and most substantial increase under hypermelatonemia to the extent of $12 \%$ and $22 \%$ respectively at 45 and 90 days [23]. However, HPOT + MT in the present study reveals further increase to the tune of $29 \%$ and $38 \%$ respectively at 45 and 90 days. Clearly, both neonatal hypothyroidism and hypermelatonema program for a definitive up regulation of folliculogenesis. Nevertheless, the qualitative aspect of folliculogenesis seems to be poorer when compared to melatonin programmed rats as, the percentage of atretic follicles is much higher in HPOT $+\mathrm{MT}$ compared to melatonin programmed rats $(22 \%$ versus $1.5 \%$ at 90 days). This is however significantly less compared to HPOT rats as the percentage of atretic follicles in HPOT rats at 90 days was $42 \%$. Further, the poorer qualitative aspect is also indicated by the lesser number of corpora lutea in HPOT + MT rats $(4.2 \%)$ which is quite similar to HPOT rats $(4.1 \%)$ as against melatonin rats $(8.1 \%)$. Obviously, the favourable deprogramming effect of MT on the quantum of early pre-

Table 4. Hormonal Status of Control and Melatonin Treated Hypothyroid Female Rats

\begin{tabular}{|c|c|c|c|c|c|c|}
\hline \multirow{2}{*}{ Treatment } & \multicolumn{3}{|c|}{ T3 } & \multicolumn{3}{|c|}{ T4 } \\
\hline & 22 days & 45 days & 90 days & 22 days & 45 days & 90 days \\
\hline $\mathrm{C}$ & $2.51 \pm 0.51$ & $3.03 \pm 0.71$ & $6.53 \pm 0.65$ & $38.0 \pm 1.3$ & $117.0 \pm 6.1$ & $236.8 \pm 25.5$ \\
\hline $\mathrm{HPOT}+\mathrm{MT}$ & $1.39 \pm 0.43$ & $5.50 \pm 0.86$ & $7.98 \pm 0.37$ & $28.2 \pm 3.7^{\mathrm{c}}$ & $268.2 \pm 8.6^{\mathrm{c}}$ & $324.4 \pm 18.5^{\mathrm{c}}$ \\
\hline
\end{tabular}

C: Control; HPOT + MT: Melatonin treated Hypothyroid. Values expressed as mean \pm SEM of six animals; ${ }^{\text {a }} P<0.01$; ${ }^{b} P<0.005$; ${ }^{c} \mathrm{P}<0.0005$. 
antral follicles is offset by the recorded increased atresia and decreased antral follicles and corpora lutea. The antagonistic effect of HPOT + MT on follicular atresia is clearly manifested in the percentage of the atretic follicles, which is lesser than the HPOT rats, but more than the MT programmed rats. As the present study has used only a smaller dose of 40 $\mu \mathrm{g}$ of melatonin/animal, it would be interesting to test the effect of higher doses of melatonin to fully offset the negative impact of hypothyroidism, and we presume that the higher dose should be successful. Since there are no studies on hypermelatonemia on a background of hypothyroidism, much less with high hypermelatonemia alone, the observations of the present study are novel and interesting needing further evaluations. The presumed favourable effects of melatonin are strengthened by the reported expression of melatonin receptor gene in the granulosa cells of developing female mice [29], the role of melatonin in regulating ovarian functions by way of progesterone production, LH receptor expression as well as GnRH and GnRH receptor gene expression [30] and, the ability of melatonin to prevent intra-follicle lipid peroxidation and provide radioprotective action to ovarian follicles $[31,32]$. The above studies demonstrated the favorable influence of melatonin on simultaneous administration schedules, while in the present study, the favourable influence is revealed as a consequence of prior neonatal administration. This is a novel observation and the mechanisms of this long lasting protection afforded by neonatal melatonin administration remains a matter of conjecture. The possible explanations could be a permanent genetic reprogramming with reference to ovarian survival/apoptotic factors and/or permanent resetting of neuroendocrine ovarian hormonal axis as part of adult plasticity changes induced by neonatal melatonin programming. With reference to the former, follicular survival or apoptosis is regulated by a number of hormones, growth factors and cytokines, which in turn activate several sub-programmes involving many genes [33]. A possible avenue of future investigations could be the understanding of the possible role of neonatal hypermelatonemia on permanent resetting of local genetic programmes resulting in the activation of survival factors and/or inhibition of apoptotic factors. In the light of reported importance of expression of oocyte GDF 9 for pre-antral follicular survival and transition to antral follicles [34], and the reported expression of FOXO 3 , an apoptotic gene, and its hypothesized up regulation by neonatal programming by hypothyroidism [35], studies on the expression of these candidate genes would be worthwhile.

The effect of HPOT + MT on the endocrine axes is marked by reduced titres of both sex hormones but increased thyroid hormone titres. The progesterone titre is intermediate to that of HPOT and MT rats suggesting the partial nullifying effect of the antagonizing actions of HPOT and MT. Both $\mathrm{T}_{3}$ and $T_{4}$ levels are significantly elevated probably indicating the potential effect of MT on $\mathrm{T}_{3}$ and HPOT on $\mathrm{T}_{4}$. Appar- ently, both thyroid hormones and melatonin have their own independent actions at the level of hypothalamus to regulate the set points of neuroendocrine thyroid and goand axes in the neonatal period.

The present study intended to examine the possibility of melatonin deprogramming the effects of thyroid deficiency in the neonatal period reports interesting but differential effects on folliculogenesis, follicular survival, ovarian weight and volume and, ovarian and thyroid hormone status. The observations suggest synergistic, cumulative or antagonistic effects of melatonin on neonatal thyroid hormone deficiency. Further investigations are warranted for proper understanding of possible adult plasticity changes induced by a hypermelatonemic status on a hypothyroid background in the neonatal period.

\section{Conflict of Interest}

There is no potential conflict of interest relevant to this article.

\section{References}

1. Chiappa SA, Fink G. Releasing factor and hormonal changes in the hypothalamic-pituitary-gonadotrophin and -adrenocorticotrophin systems before and after birth and puberty in male, female and androgenized female rats. J Endocrinol. 1977;72(2):211-224.

2. Sullivan CV, Iwamoto RN, Dickhoff WW. Thyroid hormones in blood plasma of developing salmon embryos. Gen Comp Endocrinol. 1987;65(3):337-345.

3. Kalland GA, Vera A, Peterson M, Swerdloff RS. Reproductive hormonal axis of the male rat in experimental hypothyroidism. Endocrinology. 1978;102(2):476-484.

4. Mattheij JA, Swarts JJ, Lokerse P, van Kampen JT, Van der Heide D. Effect of hypothyroidism on the pituitary-gonadal axis in the adult female rat. J Endocrinol. 1995;146(1):87-94.

5. Dijkstra G, de Rooij DG, de Jong FH, van den Hurk R. Effect of hypothyroidism on ovarian follicular development, granulosa cell proliferation and peripheral hormone levels in the prepubertal rat. Eur J Endocrinol. 1996;134(5):649-654.

6. Meisami E. Complete recovery of growth deficits after reversal of PTU-induced postnatal hypothyroidism in the female rat: a model for catch-up growth. Life Sci. 1984;34(15):1487-1496.

7. Kawada J, Mino H, Nishida M, Yoshimura Y. An appropriate model for congenital hypothyroidism in the rat induced by neonatal treatment with propylthiouracil and surgical thyroidectomy: studies on learning ability and biochemical parameters. Neuroendocrinology. 
1988;47(5):424-430.

8. Akaike M, Kato N, Ohno H, Kobayashi T. Hyperactivity and spatial maze learning impairment of adult rats with temporary neonatal hypothyroidism. Neurotoxicol Teratol. 1991;13(3):317-322.

9. Madeira MD, Cadete-Leite A, Andrade JP, Paula-Barbosa MM. Effects of hypothyroidism upon the granular layer of the dentate gyrus in male and female adult rats: a morphometric study. J Comp Neurol. 1991;314(1):171186.

10. Madeira MD, Sousa N, Lima-Andrade MT, Calheiros F, Cadete-Leite A, Paula-Barbosa MM. Selective vulnerability of the hippocampal pyramidal neurons to hypothyroidism in male and female rats. J Comp Neurol. 1992;322(4):501-518.

11. Akaike M, Kato N. Abnormal behavior, spatial learning impairment and neuropeptides caused by temporary neonatal hypothyroidism. Recent Res Dev Neuroendocrinol. 1997; 10:39-48.

12. Dufty AM, Clobert J, Moller AP. Hormones, developmental plasticity and adaptation. Trends Ecol Evol. 2002; 17: 190-196.

13. Thakkar BP, Zala VM, Ramachandran AV. Neonatal thyroid hormone deficiency programs for reduced body and ovarian weights and induces plasticity changes in adult ovarian functions. Annl Biol Res. 2011; In press.

14. Wurtman RJ, Axelrod J, Chu EW. Melatonin, a pineal substance: effect on the rat ovary. Science. 1963;141:277278.

15. Motta M, Fraschini F, Martini L. Endocrine effects of pineal gland and of melatonin. Proc Soc Exp Biol Med. 1967;126(2):431-435.

16. Debeljuk L. Effect of melatonin on the gonadotrophic function of the male rat under constant illumination. Endocrinology. 1969;84(4):937-939.

17. Kinson GA, Peat F. The influences of illumination, melatonin and pinealectomy on testicular function in the rat. Life Sci I. 1971;10(5):259-269.

18. Kinson GA, Robinson S. Gonadal function of immature male rats subjected to light restriction, melatonin administration and removal of the pineal gland. J Endocrinol. 1970;47(3):391-392.

19. Lang U, Aubert ML, Conne BS, Bradtke JC, Sizonenko PC. Influence of exogenous melatonin on melatonin secretion and the neuroendocrine reproductive axis of intact male rats during sexual maturation. Endocrinology. 1983;112(5):1578-1584.

20. Lang U, Aubert ML, Rivest RW, Vinas-Bradtke JC, Sizonenko PC. Daily afternoon administration of melatonin does not irreversibly inhibit sexual maturation in the male rat. Endocrinology. 1984;115(6):2303-2310.

21. Weaver DR. Roles of melatonin in development. In: Melatonin after four decades (eds), James Olcese. Kluwer Academic/Plenum publishers, New York. 2000.
22. Patel MM, Ramachandran AV. Time of administration of melatonin and the effects on organ weights and metabolic physiology in preweanling rat neonates. J Reprod Biol Com Endcrinol. 1992; 4:63-70.

23. Thakkar BP, Ramachandran AV. Neonatal melatonin treatment has favorable quantitative and qualitative influence on adult ovarian functions in rat. Arc Appl Sci Res. 2011; 3:180-191.

24. Plowchalk DR, Smith BJ, Mattison DR. Assessment of Toxicity to the ovary using Follicle quantitation and morphometrics. In: Methods in Toxicology. Vol. 3B. Academic Press Inc. 1993; pp:57-66.

25. Tilly JL. Ovarian follicle counts--not as simple as 1, 2, 3. Reprod Biol Endocrinol. 2003;1:11.

26. Lagu SK, Bhavsar NG, Sharma RK, Ramachandran AV. Neonatal hypothyroidism-induced changes in rat testis size, dependence on temperature. Neuro Endocrinol Lett. 2005;26(6):780-788.

27. Hess RA, Cooke PS, Bunick D, Kirby JD. Adult testicular enlargement induced by neonatal hypothyroidism is accompanied by increased Sertoli and germ cell numbers. Endocrinology. 1993;132(6):2607-2613.

28. Simorangkir DR, de Kretser DM, Wreford NG. Increased numbers of Sertoli and germ cells in adult rat testes induced by synergistic action of transient neonatal hypothyroidism and neonatal hemicastration. J Reprod Fertil. 1995;104(2):207-213.

29. Lee CJ, Do BR, Lee YH, Park JH, Kim SJ, Kim JK, Roh SI, et al. Ovarian expression of melatonin Mel(1a) receptor mRNA during mouse development. Mol Reprod Dev. 2001;59(2):126-132.

30. Woo MM, Tai CJ, Kang SK, Nathwani PS, Pang SF, Leung PC. Direct action of melatonin in human granulosa-luteal cells. J Clin Endocrinol Metab. 2001;86(10):4789-4797.

31. Kim JK, Lee CJ. Effect of exogenous melatonin on the ovarian follicles in gamma-irradiated mouse. Mutat Res. 2000;449(1-2):33-39.

32. Takasaki A, Nakamura Y, Tamura H, Shimamura K, Morioka $\mathrm{H}$. Melatonin as a new drug for improving oocyte quality. Repro Med Biol. 2003; 2:139-144.

33. Markstrom E, Svensson E, Shao R, Svanberg B, Billig H. Survival factors regulating ovarian apoptosis -dependence on follicle differentiation. Reproduction. 2002;123(1):23-30.

34. Kobayashi N, Orisaka M, Cao M, Kotsuji F, Leader A, Sakuragi N, Tsang BK. Growth differentiation factor-9 mediates follicle-stimulating hormone-thyroid hormone interaction in the regulation of rat preantral follicular development. Endocrinology. 2009;150(12):5566-5574.

35. Matsuda F, Inoue N, Maeda A, Cheng Y, Sai T, Gonda H, Goto Y, et al. Expression and function of apoptosis initiator FOXO3 in granulosa cells during follicular atresia in pig ovaries. J Reprod Dev. 2011;57(1):151-158. 